\title{
Optimization Model of Engineering Specifications Based on Grey Quality Gain-Loss Function
}

\author{
Bo Wang ${ }^{1,2,3}$, Qi Yang ${ }^{1}$, Chen Liu ${ }^{4, *}$, Qikai Li ${ }^{1}$ and Xiangtian Nie ${ }^{1,2,3,4}$ \\ 1 School of Water Conservancy, North China University of Water Resources and Electric Power, \\ Zhengzhou 450046, China; wangbo@ncwu.edu.cn (B.W.); 201703219@stu.ncwu.edu.cn (Q.Y.); \\ 201403516@stu.ncwu.edu.cn (Q.L.); niexiangtian@ncwu.edu.cn (X.N.) \\ 2 Henan Collaborative Innovation Center for Water Efficient Utilization and Guarantee Engineering, \\ Zhengzhou 450046, China \\ 3 Henan Province Key Laboratory of Water Environment Simulation and Treatment, \\ Zhengzhou 450046, China \\ 4 School of Management and Economics, North China University of Water Resources and Electric Power, \\ Zhengzhou 450046, China \\ * Correspondence: x201810105116@stu.ncwu.edu.cn
}

Citation: Wang, B.; Yang, Q.; Liu, C.; Li, Q.; Nie, X. Optimization Model of Engineering Specifications Based on Grey Quality Gain-Loss Function. Coatings 2021, 11, 1327. https:// doi.org/10.3390/coatings11111327

Academic Editor: Chi-wai Kan

Received: 9 September 2021

Accepted: 25 October 2021

Published: 29 October 2021

Publisher's Note: MDPI stays neutral with regard to jurisdictional claims in published maps and institutional affiliations.

Copyright: (c) 2021 by the authors. Licensee MDPI, Basel, Switzerland. This article is an open access article distributed under the terms and conditions of the Creative Commons Attribution (CC BY) license (https:// creativecommons.org/licenses/by/ $4.0 /)$.

\begin{abstract}
In view of the fact that the target values of some quality characteristics are grey, the grey quality gain-loss function model was applied in the analysis of the quality characteristics. At the same time, based on the analysis of engineering specifications and process capability, an optimization model of engineering specifications was proposed to minimize the expected total loss of each product and maximize the expected compensation with inspection costs, scrap costs and grey quality gain-loss into consideration. The optimal engineering specification can be obtained by using the optimization model. Through the example analysis and its application in dam concrete construction, the practicability of the model is verified, which provides an important reference for the research of the new theory of dam concrete construction quality control.
\end{abstract}

Keywords: grey quality gain-loss; engineering specifications; process capability

\section{Introduction}

According to Shewhart, one of the most important tasks in quality control is to understand and evaluate the needs and expectations of customers, and ultimately to provide products and services that meet or exceed those needs and expectations [1]. Therefore, the first step in meeting the needs of customers is to make the physical characteristics of the products meet the satisfied standards. The second step is to determine the way and the method of setting the standards for the physical characteristics of products, understand and evaluate the needs and expectations of the customers, and to provide products and services that meet or exceed those needs and expectations.

A quality loss function is proposed by Taguchi to determine the ideal target value (parameter design) and to evaluate the loss caused by deviation from the target value [2]. Because parameter design reduces performance variation by reducing the influence of the sources of variation rather than by controlling them, it is a very cost-effective technique for improving product quality. Kackar introduces the concepts of off-line quality control and parameter design and then discusses the Taguchi Method for conducting parameter design experiments [3,4]. Taguchi's philosophy recognizes the importance of both off-line and on-line quality control in reducing variation and its effects to the minimum, and covers the entire engineering and manufacturing process. Barker looks at key topics such as loss function, performance variations, signal to noise, system design and parameter and tolerance design [5].

The concept of quality loss function focuses on the evaluation of quality losses caused by deviation of quality characteristics from the target value, while the goal of quality 
planning is to minimize the total loss of producers and consumers for society; Kapur and Wang have described the use of the normal and log-normal quality characteristics to design the speciation limits based on Taguchi's quality loss function [6]. On this basis, Kapur proposed an optimization model to develop the acceptable specifications or tolerance limits. Using this model, the total quality costs caused by variation (which is always present within any acceptable limits), inspection (measurement to determine the nonconforming units) and costs related to nonconforming units (scrap or rework) can be minimized [7].

Considering the multivariate normal distribution of quality characteristics. The specification region is given by truncating the multivariate normal distribution. Kapur and Cho present the optimization model to develop the specification region for multiple quality characteristics based on the framework of multivariate quality loss function [8]. Chatterjee and Chakraborty have studied the expression of process capability index of some unilateral specification limits and the relationship between the expression and the defect rate [9]. The control chart of process capability is designed and used to evaluate the process capability in the entire production cycle.

Chen proposes the topic of selecting lower specification limits under Taguchi quality loss function [10]. Given that product quality characteristics comply with exponential distribution, an improved method is proposed based on Kapur and Wang for the economic design of specification limits; Pan and Pan have modified the Kapur's model so that the economic specification limits of symmetric loss and asymmetric loss can be established [6,7,11].

Since Taguchi quality loss function cannot describe the quality compensation effect in production practice, the concept of quality gain-loss function is proposed, and the quality gain-loss transfer model and the tolerance optimization method of quality characteristics are studied with the foundation that the constant term in Taylor series expansion is given the meaning of quality compensation. When the loss of linear term cannot be ignored and the compensation amount is constant, the quality gain-loss function model with larger-thebetter and smaller-the-better expectation is designed. Considering the compensation effect of quality characteristics in dam concrete construction, the tolerance optimization model of dam concrete construction quality is constructed, and the optimal tolerance adjustment rate of the characteristic per unit quality in each stage of the dam concrete construction is studied, so as to improve the overall construction quality to the greatest extent [12-14]. On this basis, an inverted normal quality gain-loss function based on functional characteristics of the inverted normal function is proposed, aiming at the issue that the existing quality gain-loss function cannot describe the unbounded problem in engineering practice, and the multivariate quality gain-loss function and quality gain-loss function with larger-thebetter, smaller-the-better characteristics are designed. In this paper, fuzziness of quality characteristics was analyzed, the fuzzy quality gain-loss function model was constructed and the optimal process mean for the asymmetric fuzzy quality gain-loss function was designed $[15,16]$.

\section{Grey Quality Gain-Loss Function}

Intuition and judgment play an important role when determining the target value of some quality characteristics, and some factors related to personal needs are also relevant. For this kind of quality characteristics, the determination of the target value is grey, so the application of Grey System Theory is adopted to expand the inverted normal quality gainloss model. With the following methods, the grey quality gain-loss function is constructed.

Assuming that the target value $y_{0}$ of a quality characteristic $y$ is grey, it can be expressed as grey number $\otimes \in\left[T_{1}, T_{2}\right]$, where $\left[T_{1}, T_{2}\right]$ is the interval range of the grey number, in which the quality loss can be considered as 0 . In this case, the grey quality gain-loss function is defined as: 


$$
\widetilde{L}(y)= \begin{cases}\widetilde{g}(y)+A\left[1-\exp \left(-\frac{\left(y-T_{1}\right)^{2}}{2 \sigma^{2}}\right)\right], & y \in\left[-\infty, T_{1}\right) \\ \widetilde{g}(y) & y \in\left[T_{1}, T_{2}\right] \\ \widetilde{g}(y)+A\left[1-\exp \left(-\frac{\left(y-T_{2}\right)^{2}}{2 \sigma^{2}}\right)\right], & y \in\left(T_{2},+\infty\right]\end{cases}
$$

Many quality gain-losses deviating from the target value are asymmetric in engineering practice, and therefore in order to make the grey quality gain-loss function more universal and closer to the actual situation, the piecewise function theory is applied to modify it, and the asymmetric grey quality gain-loss function model is constructed. The modified asymmetric grey quality gain-loss function is

$$
\widetilde{L}(y)= \begin{cases}\widetilde{g}(y)+A_{1}\left[1-\exp \left(-\frac{\left(y-T_{1}\right)^{2}}{2 \sigma_{1}^{2}}\right)\right], & y \in\left[-\infty, T_{1}\right) \\ \widetilde{g}(y), & y \in\left[T_{1}, T_{2}\right] \\ \widetilde{g}(y)+A_{2}\left[1-\exp \left(-\frac{\left(y-T_{2}\right)^{2}}{2 \sigma_{2}^{2}}\right)\right], & y \in\left(T_{2},+\infty\right]\end{cases}
$$

where $T_{1}$ and $T_{2}$ are the upper and lower limits of interval grey number respectively, $A_{1}$ and $A_{2}$ are the maximum possible loss caused by deviation from the target value on both sides, and $\sigma_{1}^{2}$ and $\sigma_{2}^{2}$ are the shape parameters of quality gain-loss function on both sides.

\subsection{Constant Compensation}

The quality compensation is constant, i.e., $\widetilde{g}(y)=a$, and the grey quality gain-loss function of quality characteristic $y$ is

$$
\widetilde{L}(y)= \begin{cases}a+A_{1}\left[1-\exp \left(-\frac{\left(y-T_{1}\right)^{2}}{2 \sigma_{1}^{2}}\right)\right], & y \in\left[-\infty, T_{1}\right) \\ a & , y \in\left[T_{1}, T_{2}\right] \\ a+A_{2}\left[1-\exp \left(-\frac{\left(y-T_{2}\right)^{2}}{2 \sigma_{2}^{2}}\right)\right], & y \in\left(T_{2},+\infty\right]\end{cases}
$$

when $\widetilde{g}(y)=a$ (constant), the curve of grey quality gain-loss function $y$ is shown in Figure 1.

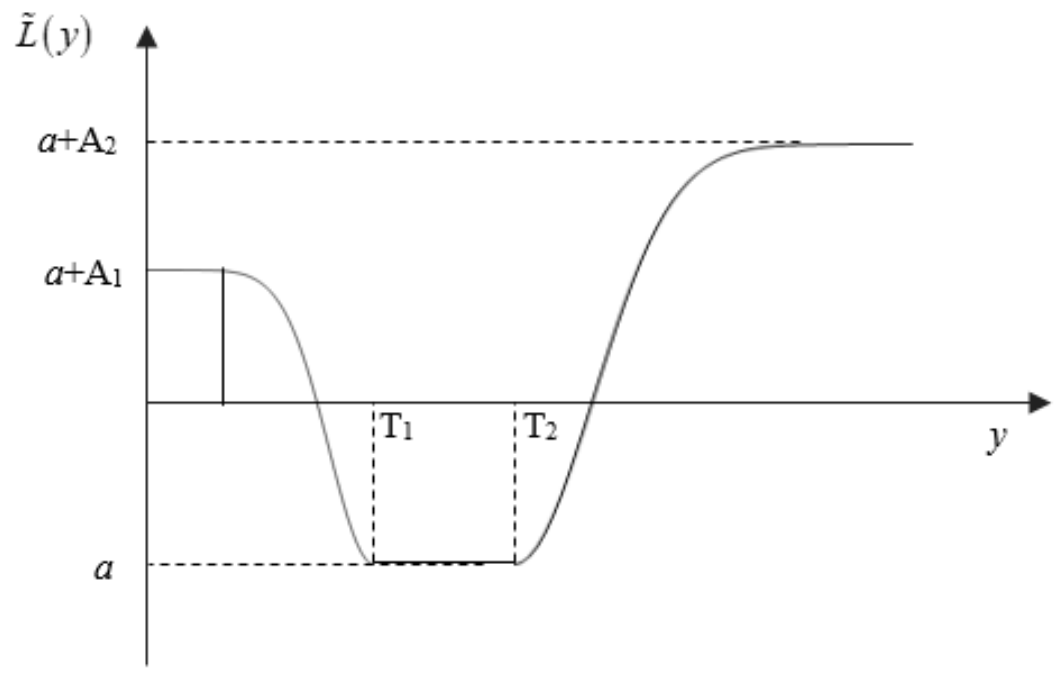

Figure 1. Curve of constant compensation.

Therefore, when the compensation is constant, the maximum grey quality compensation amount in the target value range is a. As the quality characteristics deviate from the target value, the grey quality loss increases. 


\subsection{Hyperbolic Tangent Compensation}

Furthermore, considering the variable quality compensation and the grey quality characteristic target value, if it is assumed that the target value $y_{0}$ can be expressed as the grey number $\otimes \in\left[T_{1}, T_{2}\right]$, the quality compensation will be the largest in this interval. In this case, let the quality compensation term be a function of the quality characteristic value $y$, and the grey hyperbolic tangent compensation function is constructed according to the properties of hyperbolic tangent function. At this moment, the grey quality compensation function is:

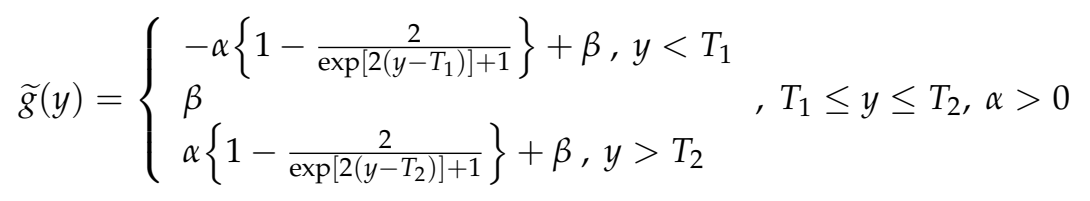

In the case of asymmetric quality compensation, the asymmetric hyperbolic tangent grey quality compensation function can be expressed as:

$$
\widetilde{g}(y)=\left\{\begin{array}{l}
-\alpha_{1}\left\{1-\frac{2}{\exp \left[2\left(y-T_{1}\right)\right]+1}\right\}+\beta, y<T_{1} \\
\beta \\
\alpha_{2}\left\{1-\frac{2}{\exp \left[2\left(y-T_{2}\right)\right]+1}\right\}+\beta, y>T_{2}
\end{array}, T_{1} \leq y \leq T_{2}, \alpha>0\right.
$$

The curve of the asymmetrical hyperbolic tangent grey quality compensation function is shown in Figure 2.

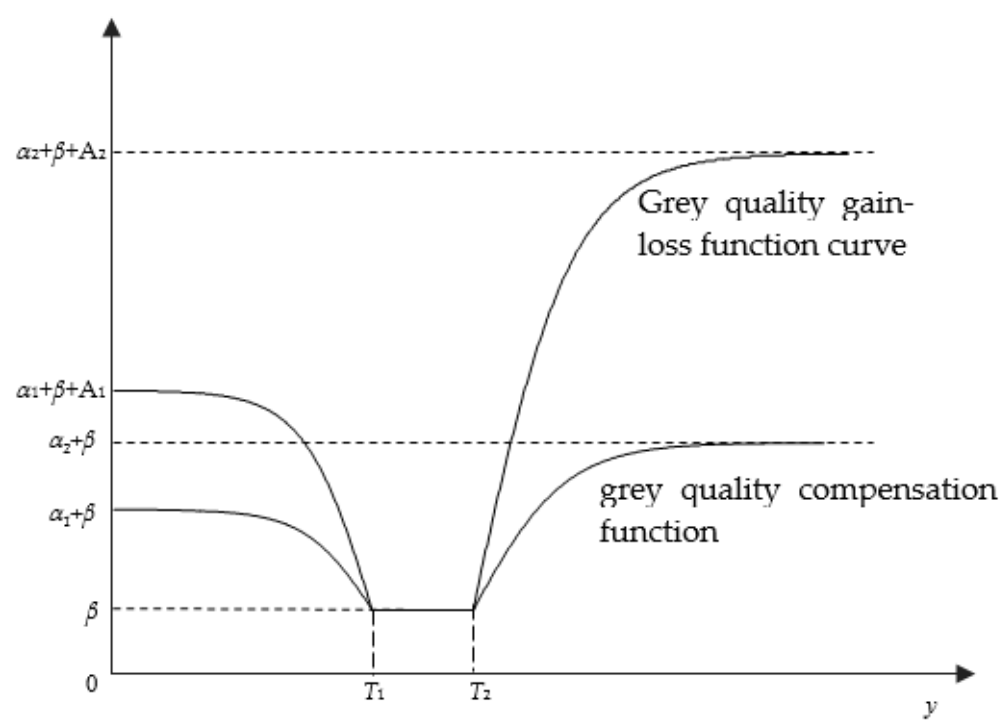

Figure 2. Comparison of grey quality function and grey gain-loss function under asymmetric hyperbolic tangent compensation $(\beta>0)$.

Therefore, the grey quality gain-loss function of asymmetric hyperbolic tangent compensation is:

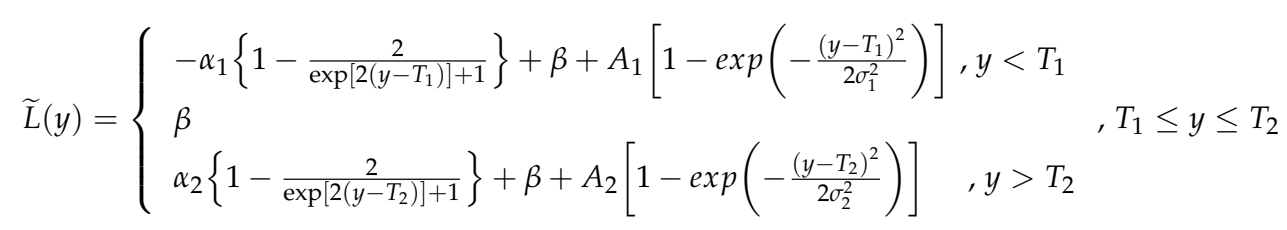

The curve of grey quality gain-loss function of asymmetric hyperbolic tangent compensation is shown in Figure 2. 
Therefore, when the compensation is hyperbolic tangent compensation, the maximum grey quality compensation in the target value interval is $\beta$. With the quality characteristic deviating from the target value, the grey quality loss increases.

\section{Process Capability Analysis}

\subsection{Process Capability}

Process capability refers to the ability of a process to ensure product quality in a stable state. Usually, process capability is expressed by six times the standard deviation $(6 \sigma)$, that is, when the process is in a stable state, $99.73 \%$ of its quality characteristic value falls within the interval of $\mu \pm 3 \sigma$, where $\mu$ is the overall mean of the quality characteristic value, and $\sigma$ is the overall standard deviation of the quality characteristic value, that is, $99.73 \%$ of the quality characteristic value falls within the $6 \sigma$ range above. Generally speaking, the smaller the value of the standard deviation is, the better it will be.

Process capability analysis is to analyze and evaluate the degree to which the process output meets the engineering standard or the technical specification proposed by customers. First, the determination and analysis of process capability is the basic work to ensure the product quality. Only when the process capability is known, can satisfied quality in the manufacturing process be controlled. If the process capability cannot meet the requirements of product design, then quality control will be impossible. Second, the measurement and analysis of process capability is an effective means to improve process capability. Through the process capability analysis, we can find the dominant factors that affect the process capability. Therefore, process capability can be increased by improving the process, equipment, operation level as well as environmental conditions. Besides, effective process methods and operation procedures, as well as strict process discipline, can be formulated to this end. Third, measurement and analysis of process capability can find out the direction for quality improvement. The actual quality status of process capability processing is the objective basis of guaranteeing product quality. Through process capability analysis, key data of process capability can be provided for designers and processes. At the same time, main problems of process capability can help find a direction to improve processing capability and product quality.

\subsection{Process Capability Index}

Process capability index is a value to measure the capacity of a process, which has been widely used in many industries. It is the main measure index to evaluate process capacity, representing the production process level and the rate of finished products in a stable production state. As for the indicators of the satisfaction degree with technical requirements, the greater the process capability index is, the better the process capability will satisfy the technical requirements, and there will even be a certain capacity reserve.

The process capability index was originally proposed by Juran [17]. If the product quality characteristics are stable and comply with normal distribution, the process capability index is defined as:

$$
C_{P}=\frac{U S L-L S L}{6 \sigma}=\frac{\Delta}{3 \sigma}
$$

Process capability index $C_{p}$ is the ratio of specification width to natural tolerance. USL is the upper limit of engineering specifications, $L S L$ is the lower limit, $\Delta=(U S L-L S L) / 2$ and $\sigma$ is the standard deviation of the process.

Since $C_{p}$ only reflects the relative relationship between the tolerance zone width and the process capability $6 \sigma$, instead of reflecting the positional relationship of the mean value $\mu$ of the process quality characteristic value with the tolerance zone, it only reflects the potential capability of the process quality to meet the technical standards. 
In order to improve the shortcomings of $C_{p}$, Kane considers the positional relationship between the mean of the process quality characteristic value and the tolerance zone, and proposes the process capability index $C_{p k}$, whose expression is as follows [18]

$$
C_{p k}=\min \left\{C_{p l}, C_{p u}\right\}=\min \left\{\frac{U S L-\mu}{3 \sigma}, \frac{\mu-L S L}{3 \sigma}\right\}=\frac{\Delta-|\mu-M|}{3 \sigma}
$$

where $M=(U S L+L S L) / 2$ is the center of the specifications and $\mu$ is the mean value of the process. $C_{p k}$ takes the average deviation of the process from the specification center into account, but it cannot reflect the deviation of the process from the target value.

Aiming at the shortcomings of $C_{p}$ and $C_{p k}$, Chan, Cheng and Spring proposes a new indicator $C_{p m}$ [19]

$$
C_{p m}=\frac{U S L-L S L}{6\left\{E(Y-T)^{2}\right\}^{\frac{1}{2}}}=\frac{\Delta}{3 \sqrt{\sigma^{2}+(\mu-T)^{2}}}
$$

where $T$ is the target value. This index is similar to Taguchi loss function. When the mean value of the process deviates from the target value, the process will face square loss.

Pearn, Kotz, and Johnson integrate $C_{p}$ and $C_{p m}$ into the index $C_{p m k}$ [20]:

$$
C_{p m k}=\frac{\min (U S L-\mu, \mu-L S L)}{3 \sqrt{E\left[(Y-T)^{2}\right]}}=\frac{\Delta-|\mu-M|}{3 \sqrt{\sigma^{2}+(\mu-T)^{2}}}
$$

Obviously, when $\mu=T=M$, the above four process capability indexes are equal. In addition, there are also process capability indicators with larger-the-better and smaller-thebetter characteristics. The summary of process capability indicators with various quality

\begin{tabular}{|c|c|c|c|c|c|}
\hline \multicolumn{2}{|c|}{ Nominal-the-Best Characteristic } & \multicolumn{2}{|c|}{ Larger-the-Better Characteristic } & \multicolumn{2}{|c|}{ Smaller-the-Better Characteristi } \\
\hline Index & Formula & Index & Formula & Index & Formula \\
\hline$C_{P}$ & $\frac{U S L-L S L}{6 \sigma}$ & $C_{P}$ & $\frac{U S L-T}{6 \sigma}$ & $C_{P}$ & $\frac{T-L S L}{6 \sigma}$ \\
\hline$C_{p k}$ & $\min \left\{\frac{U S L-\mu}{3 \sigma}, \frac{\mu-L S L}{3 \sigma}\right.$ & $C_{P u}$ & $\frac{U S L-\mu}{3 \sigma}$ & $C_{p l}$ & $\frac{\mu-L S L}{3 \sigma}$ \\
\hline$C_{p m}$ & $\frac{U S L-L S L}{3 \sqrt{\sigma^{2}+(\mu-T)^{2}}}$ & $C_{P t}$ & $\frac{\mu-T}{3 \sigma}$ & $C_{P t}$ & $\frac{T-\mu}{3 \sigma}$ \\
\hline
\end{tabular}
characteristics is shown in Table 1.

Table 1. Summary of process capability index of nominal-the-best, larger-the-better and smaller-the-better characteristics.

It can be seen from Table 1 that after the specification limit (USL, LSL) is determined, the smaller the standard deviation $\sigma$ is, the larger the process capability index and the better the process capability will be. Taking $C_{p}$ as an example, the quality level of process can be divided into five levels according to the $C_{p}$ value.

Based on the levels, corresponding judgment and disposal methods can be developed in terms of management shown in Table 2. The levels, judgment and disposal in this table are also applicable to $C_{p k}, C_{p m}$ and $C_{p m k}$. 
Table 2. Grading judgment and disposal of process capability index.

\begin{tabular}{|c|c|c|c|c|}
\hline$C_{p}$ & Level & Range & Bilateral Tolerance Range $(\mathrm{T})$ & Disposal Measures \\
\hline$C_{p}>1.67$ & Special grade & High capacity & $T \geq 10 \sigma$ & $\begin{array}{l}\text { (1) Narrow the tolerance range } \\
\text { appropriately; } \\
\text { (2) Large external fluctuations are } \\
\text { allowed to improve efficiency; } \\
\text { (3) Lower the accuracy of the } \\
\text { equipment } \\
\text { appropriately to reduce the cost; } \\
\text { (4) Make a brief test. }\end{array}$ \\
\hline $1.67 \geq C_{p}>1.33$ & First grade & Full capacity & $\mathrm{T}=8 \sigma \sim 10 \sigma$ & $\begin{array}{l}\text { (1) If is not a key product, a certain } \\
\text { degree of external fluctuations is } \\
\text { allowed; } \\
\text { (2) Simplified test; } \\
\text { (3) Control by a control chart. }\end{array}$ \\
\hline $1.33 \geq C_{p}>1$ & Second grade & $\begin{array}{l}\text { Acceptable } \\
\text { capacity }\end{array}$ & $\mathrm{T}=6 \sigma \sim 8 \sigma$ & $\begin{array}{l}\text { (1) Control by a control chart to } \\
\text { prevent } \\
\text { external fluctuations. } \\
\text { (2) For product sampling inspection, } \\
\text { pay } \\
\text { attention to the sampling method } \\
\text { and the interval. } \\
\text { (3) Inspection cannot be loosened. }\end{array}$ \\
\hline $1 \geq C_{p}>0.67$ & Third grade & $\begin{array}{l}\text { Insufficient } \\
\text { capacity }\end{array}$ & $\mathrm{T}=4 \sigma \sim 6 \sigma$ & $\begin{array}{l}\text { (1) Analyze the causes of excessive } \\
\text { range and take corresponding } \\
\text { remedial measures; } \\
\text { (2) If the final quality and assembly of } \\
\text { the product is not affected, the } \\
\text { tolerance range can be } \\
\text { appropriately expanded; } \\
\text { (3) All products are inspected or } \\
\text { screened by grades. }\end{array}$ \\
\hline$C_{p} \leq 0.67$ & Fourth grade & $\begin{array}{l}\text { Severely } \\
\text { insufficient } \\
\text { capacity }\end{array}$ & $T<4 \sigma$ & $\begin{array}{l}\text { The unqualified rate is too high, so the } \\
\text { cause should be traced immediately and } \\
\text { remedial measures should be taken to } \\
\text { improve the process capability. }\end{array}$ \\
\hline
\end{tabular}

\subsection{Improvement of Process Capability}

It can be seen from Table 1 that if the product quality needs to be improved, we must enhance the process capability. In actual production, there are usually two solutions for insufficient process capacity: the first one is to find a way of reducing the process variation and improve the stability of the process. In most cases, however, to reduce the process variation means that the production technology or construction technology needs to be greatly improved, or the production equipment needs to be updated. This method requires a lot of money, which may increase the total quality cost, and thus it has great limitations; the second method is to optimize the engineering specifications. That is, develop specification limits for the process and truncate the distribution of the quality characteristic by screening to sort out the products that do not meet the prescribed limits. From the viewpoint of manufacturers, they must decide whether screening should be performed on their products before shipping to the customer. If they decide not to screen, all the products they produce would be sent directly to the customer, and then the loss due to variability is sustained by the customer. If they decide to screen, their key concern would be how to determine the specifications for the products [21]. The formulation of engineering specifications is directly related to the qualification rate of products, costs of obsolescence and rework, and the loss of customers caused by the deviation of quality 
characteristics from the target value. Therefore, it is of great significance to formulate an appropriate specification limits in the total quality cost reduction. The improvement of engineering specifications can enhance the product quality, reduce the total quality cost and raise productivity without increasing capital investment.

Therefore, when the current process cannot be improved (i.e., decrease $\sigma^{2}$ ), one way to decrease variance of the units delivered to the customers is to add specification limits on the process and truncate the distribution by inspection. The question is how these specification limits can be developed. In quality management, engineering specifications of products are the standard to determine whether the products are qualified or not. In order to formulate the engineering specifications of products from the perspective of social and economic losses, the principle of "minimum social and economic loss" must be followed. Social and economic losses include the total cost (inspection cost, scrap (rework) cost, etc.) of an enterprise for ensuring and improving the product quality and the quality loss of the customers in the process of using the products. Specification limits are developed on the premise of minimizing the total quality cost. If the specification limits are set arbitrarily, it is likely to increase the total cost in the form of inspection. Therefore, in the product manufacturing process, improvement of engineering specifications can not only enhance the process capability, but also have an important impact on the estimation of quality loss.

\section{Improvement of the Formulation Method of Engineering Specifications}

\subsection{Shortcomings of Traditional Formulation Method of Engineering Specifications}

In the traditional quality evaluation system, if the quality characteristics of a product do not conform to the engineering specifications, the product will be deemed as unqualified, which will result in quality loss. Moreover, Taguchi believes that the society suffers losses from the initial design stage to the stage of product use due to improper product design. Kapur and Wang put forward an optimization method to determine the specification limits on the basis of minimizing the total cost or the loss of customers and manufacturers $[5,6]$. This method aims to reduce the loss borne by the customers through the inspection of online quality management, with the main idea of dividing the product quality loss into three categories, i.e., the loss suffered by customers, scrap cost or rework cost and inspection cost. Suppose that the quality characteristic $y$ conforms to the normal distribution, and the target value of quality characteristic is $y_{0}$. If the product is fully inspected, the loss costs of the quality characteristic value lower than $L S L$ and higher than USL will be scrap costs. The total quality cost under the full inspection mode is

$$
T=H(y)+(1-p) S C+I C
$$

where $H(y)$ is the expected loss per unit product suffered by the customers, SC is the scrap cost per unit product, IC is the inspection and $p$ is the qualification rate. When $\mu$ and $\sigma$ are known, the total expected loss of the product can be minimized, and the optimal solution $\eta$ can be obtained, therefore the optimal $L S L=\mu-\eta \sigma$ and the optimal $U S L=\mu+\eta \sigma$ can be calculated. With the established engineering specification limits, the products are fully inspected to screen out the products that meet the engineering specifications, and customers' loss can be reduced by truncating the normal distribution.

Traditionally, the $3 \sigma$ principle is used to determine the upper and lower limits of engineering specifications with the target value $y_{0}$ plus or minus three times the standard deviation into consideration, that is, $(L S L, U S L)=\left(y_{0}-3 \sigma, y_{0}+3 \sigma\right)$. This is because when the distribution range is $y_{0} \pm 3 \sigma$, the process achieves the maximum quality loss at the boundary of $y_{0} \pm 3 \sigma$, and the qualification probability of product quality can reach $99.73 \%$, which is close to 1 . Therefore, $\pm 3 \sigma$ or $6 \sigma$ is taken as the standard to measure whether the process capability is accurate enough and economic at the same time. However, if the engineering specifications $(L S L, U S L)$ formulated by $6 \sigma$ standard are used for the full inspection of products, the qualified rate of the products is $99.73 \%$, and thus almost all the inspected products are qualified. At this moment, the inspection cost caused by full inspection increases the total cost of products, which causes a waste of resources, and 
therefore full inspection does not exert its deserved effect. In addition, before putting forward the concept of quality compensation, measurement of quality only rests with the size of quality losses, thus the formulation of traditional optimal engineering specifications is only targeted at the quality loss function, instead of considering the role of quality compensation. As for the quality gain-loss function, inclusion of the compensation part inevitably has an impact on the engineering specifications, thus affecting the judgment on quality and the estimation of benefits. Therefore, formulation of optimal engineering specifications also requires analysis of the quality compensation part, so as to minimize the average total quality loss and maximize the average quality compensation. Therefore, formulation of engineering specifications should combine the quality loss part with the quality compensation part to optimize the formulation method of engineering specifications on the premise of minimizing the total quality loss and maximizing the quality compensation.

\subsection{Optimal Engineering Specifications under Grey Quality Loss}

In many existing literatures on formulation of engineering specifications, it is all assumed that the population distribution is normal. However, after the products are fully inspected, the quality characteristic value of the inspected products, i.e., the product delivered to the customers, is within the range of $(L S L, U S L)$. Therefore, this section assumes that the quality characteristics conform to the truncated normal distribution, and the process average is equal to the target value, that is, $(L S L, U S L)=(\mu-\eta \sigma, \mu+\eta \sigma)$. With the combination of the loss part of the grey quality gain-loss function, engineering specifications that minimize the average quality loss are formulated.

Suppose that the product quality characteristic $y$ conforms to normal distribution $Y \sim N\left(\mu, \sigma^{2}\right), F(y)$ and $f(y)$ are its distribution function and probability density function respectively. The probability density function is

$$
f(y)=\frac{1}{\sqrt{2 \pi} \sigma} \exp \left[-\frac{\left(y-y_{0}\right)^{2}}{2 \sigma^{2}}\right],-\infty \leq y \leq \infty
$$

where $\mu$ is the process mean and $\sigma^{2}$ is the variance, $-\infty \leq \mu \leq \infty, \sigma^{2} \geq 0$.

It is considered that $y$ is not less than $L S L$ and not more than $U S L$, that is, $y$ is a random variable in the interval $(L S L, U S L)$. The distribution function of the quality characteristic $y$ is:

$$
F_{T}(y)=P(Y \leq y \mid L S L \leq y \leq U S L)=\frac{P(L S L \leq Y \leq y)}{P(L S L \leq Y \leq U S L)}=\frac{F(y)-F(L S L)}{F(U S L)-F(L S L)}
$$

The probability density is

$$
f_{T}(y)=\frac{f(y)}{F(U S L)-F(L S L)}
$$

where $L S L=\mu-\eta \sigma, U S L=\mu+\eta \sigma, F(x)=\Phi\left(\frac{x-\mu}{\sigma}\right), F(U S L)-F(L S L)=\Phi(\eta)-\Phi(-\eta)=p$. Therefore, the loss of customers caused by product quality characteristics falling within the specification limits is as follows:

$$
\begin{aligned}
L_{1} & =\int_{\mu-\eta \sigma}^{T_{1}} A_{1}\left[1-\exp \left(-\frac{\left(y-T_{1}\right)^{2}}{2 \sigma_{1}^{2}}\right)\right] f_{T}(y) d y+\int_{T_{2}}^{\mu+\eta \sigma} A_{2}\left[1-\exp \left(-\frac{\left(y-T_{2}\right)^{2}}{2 \sigma_{2}^{2}}\right)\right] f_{T}(y) d y \\
& =\frac{A_{1}}{p}\left\{\begin{array}{l}
\Phi\left(\frac{T_{1}-\mu}{\sigma}\right)+\Phi(\eta)-1-\frac{\sigma_{1}}{\sqrt{\sigma^{2}+\sigma_{1}^{2}}} \times\left[\exp \left(-\frac{\left(\mu-T_{1}\right)^{2}}{2\left(\sigma^{2}+\sigma_{1}^{2}\right)}\right)\right] \times \\
{\left[\Phi\left(\frac{\sigma_{1}\left(T_{1}-\mu\right)}{\sigma \sqrt{\sigma^{2}+\sigma_{1}^{2}}}\right)-\Phi\left(\frac{\sigma\left(\mu-T_{1}\right)-\eta\left(\sigma^{2}+\sigma_{1}^{2}\right)}{\sigma_{1} \sqrt{\sigma^{2}+\sigma_{1}^{2}}}\right)\right]}
\end{array}\right\} \\
& +\frac{A_{2}}{p}\left\{\begin{array}{l}
\Phi(\eta)-\Phi\left(\frac{T_{2}-\mu}{\sigma}\right)-\frac{\sigma_{2}}{\sqrt{\sigma^{2}+\sigma_{2}^{2}}} \times\left[\exp \left(-\frac{\left(\mu-T_{2}\right)^{2}}{2\left(\sigma^{2}+\sigma_{2}^{2}\right)}\right)\right] \times \\
{\left[\Phi\left(\frac{\sigma\left(\mu-T_{2}\right)+\eta\left(\sigma^{2}+\sigma_{2}^{2}\right)}{\sigma_{2} \sqrt{\sigma^{2}+\sigma_{2}^{2}}}\right)-\Phi\left(\frac{\sigma_{2}\left(T_{2}-\mu\right)}{\sigma \sqrt{\sigma^{2}+\sigma_{2}^{2}}}\right)\right]}
\end{array}\right\}
\end{aligned}
$$


Among which, when the quality characteristics of products exceed the specification limits, the per unit scrap cost of production is:

$$
L_{2}=(1-q) S C
$$

The per unit inspection cost is IC. The total expected loss is

$$
L=L_{1}+L_{2}+I C
$$

where $\eta \geq\left(\mu-T_{1}\right) / \sigma$ and $\eta \geq\left(T_{2}-\mu\right) / \sigma, \Phi(x)$ is the function of standard normal distribution.

The Relationship between average expected loss $L$ and optimal solution $\eta$ is shown in Figure 3.

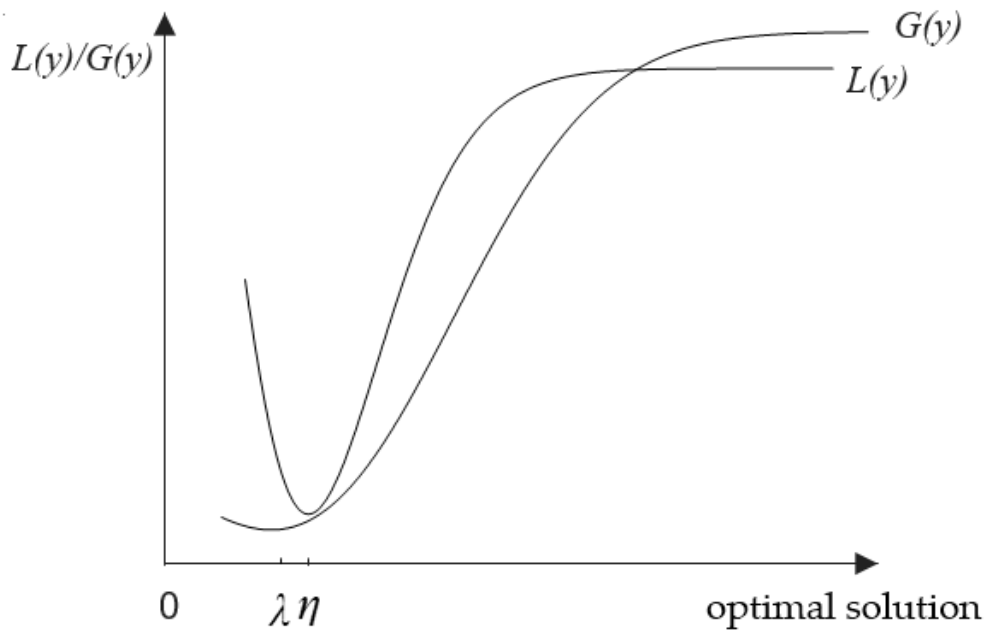

Figure 3. Relationship between average expected loss L, average expected compensation $\mathrm{G}$ and optimal solution $\eta, \lambda$.

\subsection{Optimal Engineering Specifications under Grey Quality Compensation}

For the quality gain-loss function, formulation of engineering specifications should consider not only the loss function, but also the compensation function. If the engineering specifications are developed with the traditional method, it is likely to miss the interval that maximizes the quality compensation. Therefore, formulation of the optimal engineering specifications with quality compensation must be carried out on the premise of minimizing the loss and maximizing the compensation. Optimal specifications of compensation items are collectively referred to as compensation specifications below, and the method for formulating optimal compensation specifications is explained as follows.

Calculation of the expected compensation by the hyperbolic tangent compensation function of Equation (5) is relatively complicated, therefore a function with the same variation trend can be selected to replace it when formulating the compensation specifications. It is known that the independent variable $x$ of hyperbolic tangent function is continuous on $(-\infty,+\infty)$, and $t h(x)$ has a third derivative at $x=0$, which can be expanded by Taylor series:

$$
\operatorname{th}(x)=\operatorname{th}(0)+\frac{t h^{\prime}(0)}{1 !} x+\frac{t h^{\prime \prime}(0)}{2 !} x^{2}+\frac{t h^{\prime \prime \prime}(0)}{3 !} x^{3}+o\left(x^{3}\right)
$$
$t h^{*}(x)$ :

Because $t h(0)=0, t h^{\prime}(0)=1, t h^{\prime \prime}(0)=0, t h^{\prime \prime \prime}(0)=-2, t h(x)$ can be estimated by

$$
t h^{*}(x)=x-1 / 3 x^{3}
$$

However, there is an estimation deviation between $t h^{*}(x)$ and $t h(x)$, and the variation trend between the two is consistent, that is, the interval between the maximum and minimum values is also consistent, and thus $t h^{*}(x)$ can be used to replace $t h(x)$ to construct 
the compensation function, so as to develop the compensation specifications. Therefore, quality compensation function of modified grey hyperbolic tangent can be estimated as:

$$
\widetilde{g}_{\mathcal{S}}^{*}(y)=\left\{\begin{array}{l}
-\alpha_{1}\left[\left(y-T_{1}\right)-\left(y-T_{1}\right)^{3} / 3\right]+\beta, y \leq T_{1} \\
\beta \\
\alpha_{2}\left[\left(y-T_{2}\right)-\left(y-T_{2}\right)^{3} / 3\right]+\beta, y>T_{2}
\end{array} \quad, T_{1} \leq y \leq T_{2}, \alpha>0\right.
$$

Let the lower bound of compensation specifications be $L S L^{*}=\mu-\lambda_{1} \sigma$ and the upper bound be $U S L^{*}=\mu+\lambda_{2} \sigma$, then the average expected compensation within the limits of compensation specifications is:

$$
\begin{aligned}
G & =E\left[\widetilde{g}^{*}(y)\right]=\int_{\mu-\lambda \sigma}^{\mu+\lambda \sigma} \widetilde{g}^{*}(y) f_{T}(y) d y \\
& =\frac{1}{P}\left\{\begin{array}{l}
\int_{\mu-\lambda \sigma}^{T_{1}}\left\{-\alpha_{1}\left[\left(y-T_{1}\right)-\left(y-T_{1}\right)^{3} / 3\right]+\beta\right\} f(y) d y+\int_{T_{1}}^{T_{2}} \beta f(y) d y \\
+\int_{T_{2}}^{\mu+\lambda \sigma}\left\{\alpha_{2}\left[\left(y-T_{2}\right)-\left(y-T_{2}\right)^{3} / 3\right]+\beta\right\} f(y) d y
\end{array}\right\} \\
& =\frac{1}{P}\left\{\begin{array}{l}
\beta \int_{\mu-\lambda \sigma}^{\mu+\lambda \sigma} f(y) d y-\alpha_{1}\left[\begin{array}{l}
\left(\frac{1}{3} T_{1}^{3}-T_{1}\right) \int_{\mu-\lambda \sigma}^{T_{1}} f(y) d y+\left(1-T_{1}\right)^{2} \int_{\mu-\lambda \sigma}^{T_{1}} y f(y) d y \\
+T_{1} \int_{\mu-\lambda \sigma}^{T_{1}} y^{2} f(y) d y-\frac{1}{3} \int_{\mu-\lambda \sigma}^{T_{1}} y^{3} f(y) d y
\end{array}\right] \\
+\alpha_{2}\left[\begin{array}{l}
\left(\frac{1}{3} T_{2}^{3}-T_{2}\right) \int_{T_{2}}^{\mu+\lambda \sigma} f(y) d y+\left(1-T_{2}\right)^{2} \int_{T_{2}}^{\mu+\lambda \sigma} y f(y) d y \\
+T_{2} \int_{T_{2}}^{\mu+\lambda \sigma} y^{2} f(y) d y-\frac{1}{3} \int_{T_{2}}^{\mu+\lambda \sigma} y^{3} f(y) d y
\end{array}\right]
\end{array}\right.
\end{aligned}
$$

where:

$$
\begin{gathered}
F(a)=\Phi\left(\frac{a-\mu}{\sigma}\right) \\
\int_{a}^{b} f(y) d y=\Phi\left(\frac{b-\mu}{\sigma}\right)-\Phi\left(\frac{a-\mu}{\sigma}\right) \\
\int_{a}^{b} y f(y) d y=\mu\left[\Phi\left(\frac{b-\mu}{\sigma}\right)-\Phi\left(\frac{a-\mu}{\sigma}\right)\right]+\sigma\left[\phi\left(\frac{a-\mu}{\sigma}\right)-\phi\left(\frac{b-\mu}{\sigma}\right)\right] \\
\int_{a}^{b} y^{2} f(y) d y=\left(\sigma^{2}+\mu^{2}\right)\left[\Phi\left(\frac{b-\mu}{\sigma}\right)-\Phi\left(\frac{a-\mu}{\sigma}\right)\right] \\
+[\sigma(a+\mu)+2 \mu] \phi\left(\frac{a-\mu}{\sigma}\right)-[\sigma(b+\mu)+2 \mu] \phi\left(\frac{b-\mu}{\sigma}\right) \\
\int_{a}^{b} y^{3} f(y) d y=\left(\mu^{3}+3 \mu \sigma^{2}\right)\left[\Phi\left(\frac{b-\mu}{\sigma}\right)-\Phi\left(\frac{a-\mu}{\sigma}\right)\right] \\
+\left\{\sigma^{2}\left[(a-\mu)^{2}+2 \sigma^{2}\right]+3 \mu \sigma^{2}(a-\mu)+3 \mu^{2} \sigma^{2}\right\} \phi\left(\frac{a-\mu}{\sigma}\right) \\
-\left\{\sigma^{2}\left[(b-\mu)^{2}+2 \sigma^{2}\right]+3 \mu \sigma^{2}(b-\mu)+3 \mu^{2} \sigma^{2}\right\} \phi\left(\frac{b-\mu}{\sigma}\right)
\end{gathered}
$$

where $\Phi(x)$ and $\phi(x)$ are the distribution function and probability density of standard normal distribution, respectively. Obviously, when $\mu$ and $\sigma$ are known, $G$ is a function of $\lambda$. When $\beta<0$, the quality compensation will be positive compensation, which means that the quality compensation is opposite to the quality loss. If the quality loss is always greater than 0 , the quality positive compensation will always be less than 0 , and the smaller the quality compensation is, the greater its absolute value will be, indicating that the more the positive compensation is, the better the quality will be. When $\beta>0$, it is negative compensation, and like the quality loss, it will always be positive. The smaller the value is, the less the negative compensation and the better the quality will be. In conclusion, an economic engineering specification which minimizes the average total expected compensation $G$ per unit product should be developed, i.e., $\operatorname{Min}\{G(\lambda)\}$. Through MATLAB programming calculation, the optimum solution corresponding to $\operatorname{Min}\{G(\lambda)\}$ is $\lambda$, and the optimal compensation specification $\left(L S L^{*}, U S L^{*}\right)=(\mu-\lambda \sigma, \mu+\lambda \sigma)$ can be obtained.

The Relationship between average expected compensation $G$ and optimal solution $\lambda$ is shown in Figure 3. 
If the minimum expected loss and the maximum expected compensation are required at the same time, the intersection of the optimal loss specification and the optimal compensation specification should be taken, that is $(L S L, U S L)=\left\{\max \left(L S L, L S L^{*}\right), \min \left(U S L, U S L^{*}\right)\right\}$.

\section{Example Calculation}

In the heightening project of Danjiangkou dam, the heightening construction scope of overflow dam section includes the heightening construction of 11 dam sections from overflow 14 to 24 . The construction process involves the concrete pouring of the weir surface, the second-phase concrete construction of the gate slot, the planting of steel bars and the installation of steel bars. This study took the second-phase concrete construction of the gate slot as an example to conduct research on the formulation of optimal engineering specifications based on the acceptance evaluation form of construction quality through the inspection process of general concrete appearance quality from the Construction Quality Acceptance and Evaluation Form and Formfilling Instructions for Unit Works of Water Conservancy and Hydropower Projects, edited by the Construction and Management Department of the Ministry of Water Resources, with the combination of design specifications and design requirements. In the construction quality acceptance and evaluation of the general concrete appearance quality inspection process, the surface flatness of general items was inspected. The maximum deviation per $3 \mathrm{~m}$ was $10.75 \mathrm{~mm}$ and the minimum deviation was $9.25 \mathrm{~mm}$, which met the quality requirements in the Construction Quality Acceptance and Evaluation Form and Formfilling Instructions for Unit Works of Water Conservancy and Hydropower Projects.

Therefore, $[9.25,10.75]$ can be taken as the range of target value and denoted as interval grey number $\otimes \in\left[T_{1}, T_{2}\right]$, where $T_{1}=9.25$ and $T_{2}=10.75$; according to the design requirements and engineering practice, the surface evenness $\mathrm{Y}$ is a continuous random variable and it conforms to normal distribution, with mean value $\mu=10$ and variance $\sigma^{2}=2^{2}$, i.e., $Y \sim N\left(10,2^{2}\right)$. When the measured value of quality characteristics deviates from the target value, the maximum loss can be $A=11.25$, the per unit scrap cost $S C=3$, the per unit inspection cost $I C=0.2$, compensation coefficient $\alpha=2$ and maximum compensation $\beta=-15$.

The process causes the largest loss at $T_{1}-3 \sigma$ and $T_{2}+3 \sigma$, where $T_{1}-3 \sigma=9.25-$ $3 \times 2=3.25, T_{2}+3 \sigma=10.75+3 \times 2=16.75$, adjustment coefficient $\sigma_{1}=\sigma_{2}=\sigma_{L}$, $\sigma_{1}=\frac{(9.25-3.25)}{4}=1.5, \sigma_{2}=\frac{(16.75-10.75)}{4}=1.5$.

Substitute $A=11.25, \sigma=0.5, \sigma_{L}=0.375$ and $\left(T_{1}, T_{2}\right) \in(9.25,10.75)$ into Equation (11). The total expected loss of quality per unit product is:

$$
\begin{aligned}
& L=L_{1}+L_{2}+I C \\
& =\frac{11.25}{2 \Phi(\eta)-1}\left\{\begin{array}{l}
{[-2 \Phi(0.375)+2 \Phi(2.5)]} \\
-0.6 \times\left\{\begin{array}{l}
{[\exp (-0.045)] \times[\Phi(-0.225)-\Phi(0.4-1.6667 \eta)]} \\
-[\exp (-0.045)] \times[\Phi(-0.4+1.6667 \eta)-\Phi(0.225)]
\end{array}\right\}
\end{array}\right\} \\
& +(2-2 \Phi(\eta)) \times 3+0.2
\end{aligned}
$$

where:

$$
\begin{aligned}
& L_{1}=\frac{A}{p}\left\{\begin{array}{c}
{\left[\Phi\left(\frac{T_{1}-\mu}{\sigma}\right)-\Phi\left(\frac{T_{2}-\mu}{\sigma}\right)-\Phi\left(\frac{\omega_{1}-\mu}{\sigma}\right)+\Phi\left(\frac{\omega_{2}-\mu}{\sigma}\right)\right]-\frac{\sigma_{L}}{\sqrt{\sigma^{2}+\sigma_{L}^{2}}} \times} \\
\left\{\begin{array}{l}
{\left[\exp \left(-\frac{\left(\mu-T_{1}\right)^{2}}{2\left(\sigma^{2}+\sigma_{L}^{2}\right)}\right)\right] \times\left[\Phi\left(\frac{\sigma_{L}\left(T_{1}-\mu\right)}{\sigma \sqrt{\sigma^{2}+\sigma_{L}^{2}}}\right)-\Phi\left(\frac{\sigma\left(\mu-T_{1}\right)-\eta\left(\sigma^{2}+\sigma_{L}^{2}\right)}{\sigma_{L} \sqrt{\sigma^{2}+\sigma_{L}^{2}}}\right)\right]} \\
+\left[\exp \left(-\frac{\left(\mu-T_{2}\right)^{2}}{2\left(\sigma^{2}+\sigma_{L}^{2}\right)}\right)\right] \times\left[\Phi\left(\frac{\sigma\left(\mu-T_{2}\right)+\eta\left(\sigma^{2}+\sigma_{L}^{2}\right)}{\sigma_{L} \sqrt{\sigma^{2}+\sigma_{L}^{2}}}\right)-\Phi\left(\frac{\sigma_{L}\left(T_{2}-\mu\right)}{\sigma \sqrt{\sigma^{2}+\sigma_{L}^{2}}}\right)\right]
\end{array}\right\}
\end{array}\right\} \\
& =\frac{11.25}{2 \Phi(\eta)-1}\left\{\begin{array}{l}
{[-2 \Phi(0.375)+2 \Phi(2.5)]} \\
-0.6 \times\left\{\begin{array}{l}
{[\exp (-0.045)] \times[\Phi(-0.225)-\Phi(0.4-1.6667 \eta)]} \\
-[\exp (-0.045)] \times[\Phi(-0.4+1.6667 \eta)-\Phi(0.225)]
\end{array}\right\}
\end{array}\right\} \\
& L_{2}=(1-p) S C=(2-2 \Phi(\eta)) \times 3
\end{aligned}
$$


Obviously, it is known that $L$ is a function of $\eta$. Next, the economic engineering specification that minimizes the average total expected loss $L$ per unit product is formulated, that is:

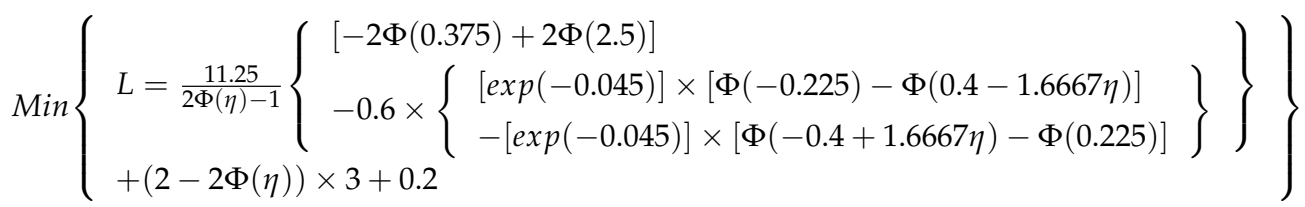

With charting and calculation by MATLAB programming, the optimal solution corresponding to $\operatorname{Min}\{L(\eta)\}$ is $\eta=0.8750$, as shown in Figure 4.

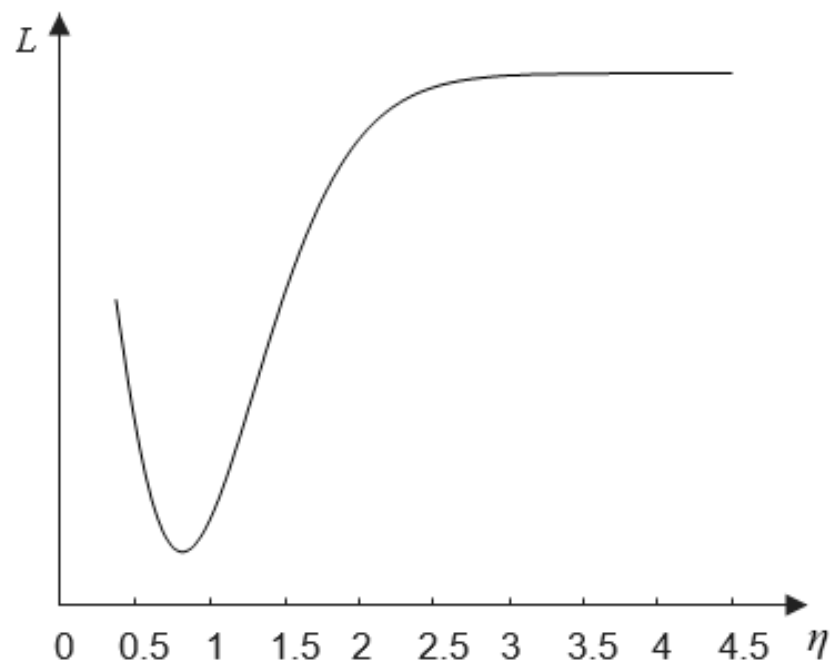

Figure 4. Relationship between average expected loss $l$ and optimal solution $\eta$.

Substitute the optimal solution into the formula $(U S L, L S L)=(\mu-\eta \sigma, \mu+\eta \sigma)$ for the upper and lower limits of the optimal engineering specification, it can be obtained that:

The lower limit of the optimal specification for the quality loss part is $L S L=\mu-\eta \sigma=$ $10-0.745 \times 2=8.51$.

The upper limit of the optimal specification for the quality loss part is $U S L=\mu+\eta \sigma=$ $10+0.745 \times 2=11.49$.

Substitute $\alpha=2, \beta=-15, A=11.25,\left(T_{1}, T_{2}\right) \in(9.25,10.75), \mu=10, \sigma=2, \sigma_{L}=1.5$, into Equation (15), we have:

$$
\begin{aligned}
G & =E\left[g^{*}(y)\right]=-15[1-2 \Phi(\lambda)] \\
& -2\left[880.0521-1523.8594 \Phi(-\lambda)+\left(483.7916-63.6667 \lambda-\frac{16}{3} \lambda^{2}\right) \phi(-\lambda)\right] \\
& +2\left[2098.6407[\Phi(\lambda)-\Phi(0.375)]-880.1667 \phi(0.375)-\left(432.4583-37 \lambda-\frac{4}{3} \lambda^{2}\right) \phi(\lambda)\right]
\end{aligned}
$$

Obviously, $G$ is a function of $\lambda . \beta<0$, so the quality compensation is positive, which means that the quality compensation value is always less than 0 , and the smaller the quality compensation value is, the larger its absolute value will be, indicating more compensation. Therefore, the economic engineering specification that minimizes the average total expected compensation $G$ per unit product is formulated, that is, $\operatorname{Min}\{G(\eta)\}$. With charting and calculation by MATLAB programming, the optimal solution corresponding to $\operatorname{Min}\{G(\eta)\}$ is $\lambda=0.69$, as shown in Figure 5. 


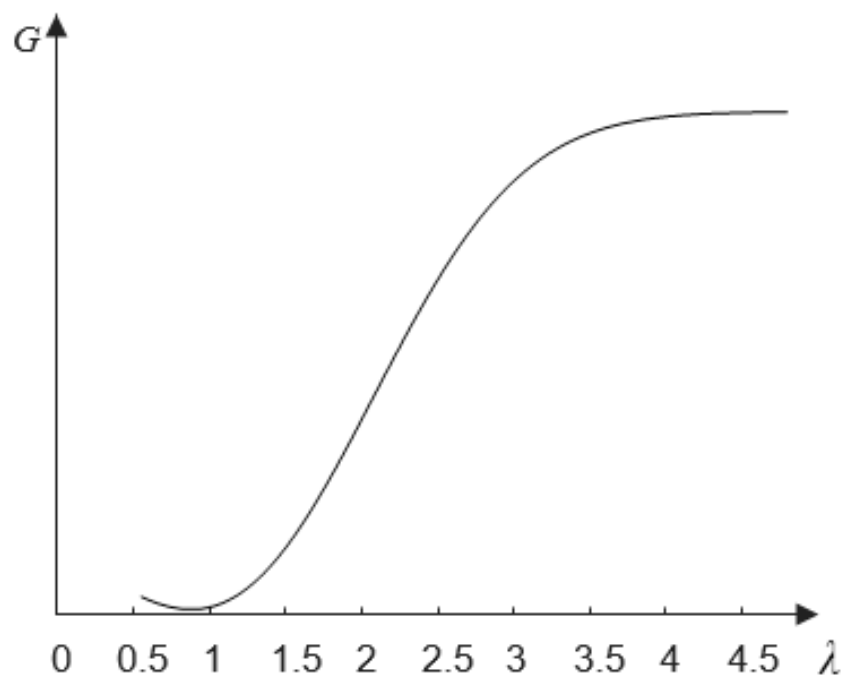

Figure 5. Relationship between average expected compensation $g$ and optimal solution $\lambda$.

By substituting the optimal solution into the formula $\left(L S L^{\prime}, U S L^{\prime}\right)=(\mu-\lambda \sigma, \mu+\lambda \sigma)$ of the upper and lower limit of the optimal compensation specification, we can get:

The lower limit of the optimal compensation specification $L S L^{*}=\mu-\lambda \sigma=10-$ $0.69 \times 2=8.62$.

The upper limit of the optimal compensation specification $U S L^{*}=\mu+\lambda \sigma=10+$ $0.69 \times 2=11.38$.

In conclusion, in order to minimize the expected loss and maximize the expected compensation, the intersection of the optimal loss specification and the optimal compensation specification shall be taken as the optimal engineering specification, i.e., $(L S L, U S L)=$ $\{\max (8.51,8.62), \min (11.49,11.38)\}=(8.62,11.38)$. The specifications of the project meet the quality requirements in the construction quality acceptance evaluation form and filling instructions of unit works of water resources and hydropower projects. Taking this section as the specification limit of the ordinary concrete appearance quality inspection process, the distribution can be cut off through inspection, which can improve the concrete appearance quality, reduce the total quality cost and improve the concrete construction efficiency without increasing capital investment.

\section{Conclusions}

Because the quality evaluation standard is grey in some cases, the calculation of quality profit and loss is also grey. The definition of some quality indicators in the actual construction and production process is also grey. Intuition and judgment play an important role in determining the target value of some quality characteristics, and some factors related to personal needs also have an effect. For this kind of quality characteristics, determination of the target value has a certain degree of greyness, therefore the grey quality gain-loss function model was established. In view of insufficient process capability, specification bounds can be imposed on the process and the distribution can be truncated with inspection if the current process cannot be improved, so as to lessen the differences of projects delivered to customers. However, the formulation of specification limits should take the minimization of the total quality cost of producers and consumers as the basis. Losses borne by producers include costs related to inspection (tests to determine defective products) and defective products (obsolescence or rework). At the same time, for the products with compensation functions, the effect of quality compensation should be considered, and the maximum quality compensation should be achieved on the premise of minimizing the total quality cost. With the optimization model proposed in this paper, optimal engineering specification limits can be obtained. Finally, taking the Danjiangkou dam heightening project as an example, in the construction quality acceptance and evaluation of the general 
concrete appearance quality inspection process, for the surface flatness of general items, the optimal engineering specification limits can be obtained by using the optimization model proposed in this paper. Without increasing capital investment, the appearance quality of concrete is improved, the total quality cost is reduced and the concrete construction efficiency is improved.

In this paper, the research is carried out on the premise that the quality characteristics obey the normal distribution. However, in engineering practice, the distribution characteristics of each quality characteristic often have a variety of situations, such as uniform distribution, exponential distribution and Weber distribution. Therefore, subsequent researchers can extend the case of non-normal distribution. For the part of compensation function, this paper only considers two forms: constant compensation and hyperbolic tangent compensation. Subsequent researchers can develop other compensation functions in line with the actual compensation situation. The multivariate grey quality gain-loss function can also be deeply discussed and analyzed.

Author Contributions: Conceptualization, B.W. and Q.Y.; methodology, B.W.; software, C.L.; validation, Q.Y. and Q.L.; formal analysis, X.N.; investigation, Q.Y.; resources, C.L.; data curation, B.W.; writing-original draft preparation, Q.Y.; writing-review and editing, C.L.; visualization, X.N.; supervision, Q.L.; project administration, B.W.; funding acquisition, B.W. All authors have read and agreed to the published version of the manuscript.

Funding: Natural Science Foundation of China (No. 51709116).

Institutional Review Board Statement: Not applicable.

Informed Consent Statement: Not applicable.

Data Availability Statement: No data supporting results.

Conflicts of Interest: The authors declare no conflict of interest.

\section{References}

1. Shewhart, W.A. Economic Control. of Quality Manufactured Product; Van Nostrand Company: New York, NY, USA, 1931.

2. Taguchi, G. Quality engineering in Japan. Communication in Statistics. Theory Methods 1985, 14, 2785-2801. [CrossRef]

3. Kackar, R.N. Off-line quality control, parameter design and the Taguchi method. J. Qual. Technol. 1985, 17, 176-188. [CrossRef]

4. Kackar, R.N. Taguchi's Quality Philosophy: Analysis and Commentary. Qual. Prog. 1989, 2, 3-21. [CrossRef]

5. Barker, T.R. Quality Engineering by Design: Taguchi's Philosophy; Taylor \& Francis: Oxfordshire, UK, 1986; pp. 37-42.

6. Kapur, K.C.; Wang, C.J. Economic Design of Specifications Based on Taguchi's Concept of Quality Loss Function. Quality Design, Planning, and Control; The American Society of Mechanical Engineers: Boston, MA, USA, 1987; pp. 23-36.

7. Kapur, K.C. An approach for development of specifications for quality improvement. Qual. Eng. 1988, 1, 63-77. [CrossRef]

8. Kapur, K.C.; Cho, B.-R. Economic design of the specification region for multiple quality characteristics. IIE Trans. 1996, 28, 237-248. [CrossRef]

9. Chatterjee, M.; Chakraborty, A.K. Some process capability indices for unilateral specification limits-Their properties and the process capability control charts. Commun. Stat. Theory Methods 2014, 45, 7130-7160. [CrossRef]

10. Chen, C.-H. Specification limit under a quality loss function. J. Appl. Stat. 1999, 26, 903-908. [CrossRef]

11. Pan, J.N.; Pan, J.B. A comparative study of various loss functions in the economic tolerance design. In Proceedings of the IEEE International Conference on Management of Innovation and Technology, Singapore, 21-23 June 2006; pp. $783-787$.

12. Wang, B.; Li, Z.; Gao, J.; Vaso, H. Critical Quality Source Diagnosis for Dam Concrete Construction Based on Quality Gain loss Function. J. Eng. Sci. Technol. Rev. 2014, 7, 137-151. [CrossRef]

13. Wang, B.; Zhou, H.G.; Li, Z.Y.; Nie, X.T. Tolerance optimization for quality characteristics of the dam concrete construction based on quality gain-loss function. Math. Pract. Theory 2016, 46, 90-99.

14. Wang, B.; Fan, T.Y.; Tian, J.; Liu, M.Q.; Nie, X.T. Designing a quality gain-loss function for larger-the-better char-acteristic and smaller-the-better characteristic under not neglecting the linear term loss and keeping compensation amount constant. Math. Pract. Theory 2019, 49, 153-160.

15. Nie, X.T.; Liu, C.; Wang, B. Inverted normal quality gain-loss function and its application in water project con-struction. J. Coast. Res. 2020, 104, 415-420. [CrossRef]

16. Nie, X.T.; Liu, C.; Guo, W.J.; Liu, M.Q.; Wang, B. Fuzzy quality gain-loss function model and process mean design optimization. Henan Sci. 2020, 38, 1377-1386.

17. Ireson, W.G.; Juran, J.M. Quality-Control Handbook. J. Am. Stat. Assoc. 1952, 47, 317. [CrossRef]

18. Kane, V.E. Process capability indices. J. Qual. Technol. 1986, 18, 41-52. [CrossRef] 
19. Chan, L.K.; Cheng, S.W.; Spiring, F.A. A New Measure of Process Capability:Cpm. J. Qual. Technol. 1988, 20, 162-175. [CrossRef]

20. Pearn, W.L.; Kotz, S.; Johnson, N.L. Distributional and Inferential Properties of Process Capability Indices. J. Qual. Technol. 1992, 24, 216-231. [CrossRef]

21. Kapur, K.; Cho, B.-R. Economic Design and Development of Specifications. Qual. Eng. 1994, 6, 401-417. [CrossRef] 